\title{
Alimentación de dos especies de anfibios (Anura: Hylidae) en la estación de bajas temperaturas y su relación con la acumulación de energía en Santa Fe, Argentina
}

\author{
Carolina Elizabet Antoniazzi ${ }^{1,2}$, Javier Alejandro López ${ }^{1,2}$, Marta Duré ${ }^{3}$ \& Diego Alejandro Falico ${ }^{2}$ \\ 1. Universidad Nacional del Litoral, Facultad de Humanidades y Ciencias, Departamento de Ciencias Naturales, Ciudad \\ Universitaria, Paraje el Pozo S/N (3000), Santa Fe, Argentina; caroantoniazzi@gmail.com \\ 2. Instituto Nacional de Limnología (CONICET-UNL), Ciudad Universitaria, Paraje el Pozo S/N (3000), Santa Fe, \\ Argentina; jalopez@inali.unl.edu.ar, dialefalico@gmail.com \\ 3. Centro de Ecología Aplicada del Litoral (CONICET), Ruta Provincial 5, Kilómetro 2,5 (291), Corrientes, Argentina; \\ martadure@yahoo.com
}

Recibido 19-IV-2012. Corregido 30-VIII-2012. Aceptado 25-IX-2012.

\begin{abstract}
Feeding of two amphibian species (Anura: Hylidae) during the low temperatures season and its relationship with energy storage in Santa Fe, Argentina. In environments with thermal and pluvial seasonality such as those of the Middle Paraná River floodplain (Province of Santa Fe, Argentina), most amphibian species reproduce during the warm season and drastically diminish their activity during winter. Even though, a few species remain active during the cold season, such as Hypsiboas pulchellus that has its reproductive peak during the autumn-winter period (and the consequent energy demand). The objective of this study was to analyze and compare the feeding and development of fat bodies during the low temperature season for H. pulchellus and Dendropsophus nanus. We analyzed entire gastrointestinal tract contents of both species (H. pulchellus=110 specimens; D. nanus $=114$ specimens) and applied an index (IRI\%) that combines prey abundance, volume and frequency to describe frogs diets; we used fat bodies weights as indicators of stored energy reserves. We compared diet between species with a niche overlap index (Ojk: 0-1) and used null models to ascribe statistical significance to evaluate overlap; and we analyzed variation in empty guts proportions through months and between species. Also, using ANCOVAs we explored differences in fat bodies, number and volume of prey consumed along months, between species and sexes. The most important preys in $H$. pulchellus diet during the cold season were Araneae (IRI\%=34.96), Chironomidae (IRI\%=33.08), Tipulidae $($ IRI $\%=11.44)$ and Gryllidae $($ IRI $\%=7.31)$; while for $D$. nanus, Chironomidae (IRI\%=48.14), Tipulidae (IRI\%=18.41), Psychodidae (IRI\%=7.44) and Araneae $(\mathrm{IRI} \%=7.34)$. Diet overlap between species was elevated $(\mathrm{Ojk}=0.78)$ and higher than expected by chance (mean simulated indices: Ojk=0.04; p [observed $\geq$ expected $]<0.01 ; \mathrm{p}[$ observed $\leq$ expected $]=1$ ). In $H$. pulchellus there was a monthly variation in number of prey per gut, while in $D$. nanus there was a difference in fat bodies development between sexes. Fat bodies development, number of prey per gut and preys volume also varied between species. Despite diet similarity between H. pulchellus and D. nanus, each species showed a different strategy to accumulate energy and support their activity during the cold season. The low rate of gastrointestinal emptiness in $H$. pulchellus ( $<10 \%$ in any analyzed month) together with the poor development of their fat bodies, allows us to point out that, to sustain the breeding elevated energy demands, this species continues with a high feeding rate even at the low temperatures of cold season. On the other hand, the rate of gastrointestinal emptiness of D. nanus was higher than that of $H$. pulchellus (May $=17.24 \%$, July $=22.22 \%$ and August $=35.71 \%$ ), while their fat bodies were well developed. Thus, $D$. nanus would depend more on their stored reserves to sustain the energy demands of being active during the low temperatures season and hence would reach the reproductive season in spring-summer in good body condition. Rev. Biol. Trop. 61 (2): 875-886. Epub 2013 June 01.
\end{abstract}

Key words: Hypsiboas pulchellus, Dendropsophus nanus, diet, fat bodies, cold season, energy source. 
En las regiones de climas con estacionalidad térmica y pluviométrica, la mayoría de las especies de los ensambles locales de anfibios se alimentan y reproducen en la estación cálida y lluviosa (Sánchez et al. 2007, López et al. 2011). Sin embargo, en los meses más fríos, la mayoría de las especies entran en hibernación, disminuyendo drásticamente la actividad de sus poblaciones (López et al. 2011). Las bajas temperaturas inducen a bajas tasas metabólicas, y los cuerpos grasos son la principal fuente de energía acumulada para sobrevivir durante varios meses (Prado \& Hadad 2005, Wells 2007). No obstante, en los ambientes subtropicales del río Paraná Medio muchos anfibios poseen un período de actividad muy prolongado (Iriondo et al. 2007), el cual abarca no solo la fase reproductiva (casi exclusivamente durante la estación cálida) sino que se extiende durante gran parte del año, incluso pueden permanecer en actividad una vez pasado el otoño (López et al. 2011).

Pese a esto, los estudios sobre alimentación de anfibios se han centrado principalmente en la estación cálida (e.g. Basso 1990, Peltzer \& Lajmanovich 2000, Duré \& Kehr 2004, López et al. 2007, 2009) y solo algunos pocos han tenido en cuenta la estación fría (Maneyro \& da Rosa 2004, Guimarães et al. 2011). Esta situación ha derivado en escasa información sobre lo que ocurre con la alimentación de aquellas especies activas durante la estación fría o las estrategias de utilización de reservas lipídicas como fuente de energía durante el periodo de bajas temperaturas.

Los anfibios activos durante el periodo otoño-invierno son sometidos a presiones selectivas dadas por las bajas temperaturas, por ejemplo, afectando la disponibilidad y calidad de alimentos (Wunder 1984). Si bien la mayoría de las especies que habitan el litoral fluvial Argentino poseen una reproducción restringida a la primavera-verano (Sánchez et al. 2007, López et al. 2011), existen algunas que pueden reproducirse durante la estación de bajas temperaturas (Sánchez et al. 2007). Estas especies deberían contar con una estrategia de obtención de energía (alimentación y reservas energéticas) distinta a las empleadas por aquellas que, si bien continúan en actividad durante el otoño-invierno, no precisan la elevada cantidad de energía que demanda el evento reproductivo. Por tal motivo, el objetivo de éste trabajo fue analizar y comparar la alimentación y el desarrollo de los cuerpos grasos durante la temporada de bajas temperaturas de Hypsiboas pulchellus y Dendropsophus nanus, en ambientes de la planicie de inundación del río Paraná Medio.

\section{MATERIALES Y MÉTODOS}

\section{Área de estudio y captura de los ejem-} plares: Los muestreos se llevaron a cabo en la isla Sirgadero y la Reserva de la Ciudad Universitaria de la Universidad Nacional del Litoral (3140'18.20” S - 60³7’04.37” W), ambientes ubicados en las cercanías de la ciudad de Santa Fe (Provincia de Santa Fe, Argentina) y correspondientes a la planicie aluvial del río Paraná Medio. La región presenta un clima templado, subhúmedo-húmedo, mesotermal, con regímenes pluviales con una marcada estación lluviosa en verano (Panigatti et al. 1981). Las medias de temperaturas del área de estudio registradas en un período de 8 años (2004-2012) fueron para la estación cálida (noviembre-marzo): media temperatura máxima $31.16 \pm 2.63^{\circ} \mathrm{C}$, media mínima $19.68 \pm 1.33^{\circ} \mathrm{C}$; y para la estación fría (abril-octubre): media temperatura máxima $21.24 \pm 3.23^{\circ} \mathrm{C}$, media mínima $11.71 \pm 2.27^{\circ} \mathrm{C}$. La media de la temperatura mínima para dicho período registradas en el mes más frío (julio) fue de $9.23 \pm 3.72^{\circ} \mathrm{C}$, mientras que la media de la temperatura máxima para el mes más cálido (enero) fue $34.94 \pm 4.08^{\circ} \mathrm{C}$. Las temperaturas máxima y mínima registradas para el período muestreado (de mayo a septiembre 2005) fueron en promedio $28.71 \pm 0.62^{\circ} \mathrm{C}$ y $3.42 \pm 1.79^{\circ} \mathrm{C}$, respectivamente (Centro de Informaciones Meteorológicas, Facultad de Ingeniería y Ciencias Hídricas, Universidad Nacional del Litoral).

En el lapso comprendido entre mayo y septiembre 2005 se recolectaron 110 especímenes adultos de $H$. pulchellus (103 machos 
y 7 hembras) y 114 especímenes adultos de D. nanus (55 machos y 59 hembras). La captura de los anfibios se realizó mensualmente en la reserva universitaria y en cinco cuerpos de agua de la isla Sirgadero. A mediados de cada mes se visitó un sitio de muestreo por día, hasta completar los seis ambientes seleccionados. Cada mes, en cada ambiente, se realizó un esfuerzo de captura manual de dos horas a partir del crepúsculo. Los especímenes capturados fueron inmediatamente sacrificados mediante la aplicación de un anestésico de uso humano (benzocaína) sobre la piel de la cabeza $\mathrm{y}$ vientre, y fijados in situ con una solución al $10 \%$ de formalina amortiguada en el transcurso de no más de una hora posterior a la eutanasia (McDiarmid 1994, Angulo et al. 2006). Los ejemplares se encuentran depositados en la colección de referencia del Laboratorio de Herpetología del Instituto Nacional de Limnología (INALI: CONICET-UNL).

Metodología de laboratorio: Se midió la longitud hocico-cloaca de cada ejemplar con un calibre digital de precisión $0.01 \mathrm{~mm}$ y se registró su peso en una balanza digital de precisión $0.00001 \mathrm{~g}$.

Los cuerpos grasos son órganos especiales donde se acumulan sustancias de reserva (lípidos) y se encuentran ubicados en el extremo anterior de las gónadas (Duellman \& Trueb 1986). El peso de los cuerpos grasos varía de acuerdo con la temperatura y el alimento disponible (Jørgensen 1986), por lo tanto es un buen indicador del estado nutricional del organismo (Jørgensen 1992). Tras la disección de los ejemplares se extrajeron y pesaron los dos cuerpos grasos en una balanza digital con precisión de $0.00001 \mathrm{~g}$. Se utilizó este método debido a que los mismos poseen forma irregular, lo que dificulta una medición precisa (Vitt \& Ohmart 1975). La masa de los cuerpos grasos se utilizó para estimar las reservas energéticas de los individuos (Martori et al. 2005).

Para el análisis de la dieta, se extrajo el tracto digestivo completo de cada individuo. Posteriormente, bajo lupa binocular, se cuantificaron y clasificaron los ítems alimentarios hasta el menor nivel taxonómico posible, debido a su grado de digestión (usualmente familia). Se tomaron las medidas de longitud total y ancho máximo del cuerpo de las presas con un calibre digital de precisión $0.01 \mathrm{~mm}$. Éstas medidas fueron utilizadas para estimar el volumen usando la fórmula de esferoide (Dunham 1983, Duré \& Kehr 2004, López et al. 2007), $\mathrm{V}=4 / 3 \pi(1 / 2 \mathrm{~L})(1 / 2 \mathrm{~A})^{2}$, donde $\mathrm{V}=$ volumen de la presa; $\pi=3.14159$; $\mathrm{L}=$ longitud máxima de la presa; y $\mathrm{A}=$ ancho máximo de la presa. Se consideró la longitud y el ancho máximo del cuerpo de la presa descartando apéndices como antenas, patas, ovipositores u ornamentaciones del cuerpo tales como espinas o artejos, entre otros (Parmelee 1999). Siguiendo el criterio de Cuevas \& Martori (2007), en caso de presas desarticuladas, el tamaño original de la presa fue estimado por comparación con presas de referencia.

La contribución de cada categoría de alimento a la dieta de las especies se calculó usando tres medidas: número, volumen y frecuencia de ocurrencia para cada ítem presa, las cuales fueron combinadas utilizando el Índice de Importancia Relativa de Pinkas et al. (1971): IRI=\% FO $(\% \mathrm{~N}+\% \mathrm{~V})$, donde $\% \mathrm{FO}$ es la frecuencia de ocurrencia porcentual de la categoría de alimento en los tractos digestivos analizados, $\% \mathrm{~N}$ es el porcentaje numérico de la categoría de alimento y $\% \mathrm{~V}$ el porcentaje volumétrico de la misma. Ya que el resultado del IRI son valores sin un límite superior, para facilitar la interpretación y la realización de comparaciones se efectuó una jerarquización mediante su transformación a porcentajes (IRI\%) (Cuevas \& Martori 2007).

Debido a la diferencia en el número de ejemplares de cada especie, para poder comparar la diversidad de presas consumidas se realizó un análisis de rarefacción con el programa EcoSim 7.72 (Gotelli \& Graves 1996, Gotelli \& Entsminger 2004). En el análisis, se calculó la diversidad de Shannon-Weaver (1949) en base al logaritmo neperiano (ln). Con el programa MVSP (Versión 3.13c) se determinó la equitatividad de las presas consumidas utilizando el índice de Pielou (1966), el cual 
se calculó como la proporción de la diversidad observada en relación a la máxima diversidad esperada. Además, para evaluar la similitud de las dietas entre las dos especies se utilizó el índice simétrico de solapamiento propuesto por Pianka (1973):

$$
\mathrm{O}_{j k}=\sum_{i}^{n} \mathrm{P}_{i j} \mathrm{P}_{i k} / \sqrt{ }\left(\sum_{i}^{n} \mathrm{P}^{2} \sum_{i j}^{n} \mathrm{P}_{i k}^{2}\right) ;
$$

donde $P_{i j}$ y $P_{i k}$ representan las proporciones del recurso $i$ utilizado por las especies $j$ y $k$ respectivamente; y $n$ el número total de estados del recurso; este índice genera valores entre 0 (sin solapamiento) y 1 (con solapamiento total). El análisis se realizó con el programa EcoSim 7.72 (Gotelli \& Entsminger 2004) con base en los valores del IRI\% de las presas. Para determinar si el solapamiento observado difiere del esperado por azar, se realizó un análisis de randomización que ofrece el programa EcoSim (Gotelli \& Entsminger 2003). Este programa realiza permutaciones de Monte Carlo para crear "pseudo-comunidades" (Pianka 1974), y compara estadísticamente los patrones en estas pseudocomunidades y la matriz real de datos. Para los análisis aquí efectuados, se realizaron 1000 permutaciones con base en la matriz original de datos, reteniendo la amplitud del nicho (Duré \& Kehr 2004).

Debido a que el peso de los ejemplares (PC) y la longitud hocico-cloaca (LHC) se encuentran altamente correlacionados $(H$. pulchellus: Spearman $\mathrm{r}=0.88, \mathrm{p}=0.0001 ; D$. nanus: Pearson $\mathrm{r}=0.92, \mathrm{p}=0.0001$ ), para representar el tamaño de los anuros en los análisis multivariados se utilizó solamente el PC. Se realizaron análisis de ANCOVA con el programa SPSS 11.55 (Statistical Package for Social Sciences Incorporated, Chicago IL, USA) para comparar el peso de los cuerpos grasos (CG), el volumen (Vol) y cantidad (N) de presas ingeridas entre sexos, entre meses y entre especies, usando el PC de los ejemplares como covariable. Cuando fue necesario, para cumplir los requisitos del análisis las variables fueron transformadas mediante una de las siguientes fórmulas: $\log (\mathrm{x}+1) \circ \mathrm{x}^{2}-0.5$. Para este análisis se consideraron todos los ejemplares que poseían al menos una presa en sus contenidos gastrointestinales. Además, para el análisis entre meses se excluyó el único ejemplar de $H$. pulchellus capturado en el mes de junio y los únicos dos ejemplares de $D$. nanus capturados en septiembre. Para $H$. pulchellus no se realizaron las comparaciones entre sexos debido al escaso número de hembras $(\mathrm{n} \bigcirc=7)$.

\section{RESULTADOS}

El $78.64 \%$ de los machos de H. pulchellus $(\mathrm{n}=103)$ contenían cuerpos grasos con un peso medio de $0.0038 \pm 0.005 \mathrm{~g}$; mientras que las hembras $(\mathrm{n}=7)$ no poseían cuerpos grasos desarrollados. El peso medio de los cuerpos grasos de todos los ejemplares de $D$. nanus fue $0.0075 \pm 0.0041 \mathrm{~g}$; el de los machos $(\mathrm{n}=55)$ $0.0064 \pm 0.0034 \mathrm{~g}$ y el de las hembras $(n=59)$ $0.0085 \pm 0.0043 \mathrm{~g}$. Los resultados de los ANCOVAs sobre la variación de los cuerpos grasos entre sexo (solo para D. nanus), entre meses y entre especies se detallan en el cuadro 1.

En H. pulchellus, de los 110 ejemplares analizados, 104 contenían alimentos. En todos los meses, más del $90 \%$ de los ejemplares poseían contenidos en sus tractos digestivos (Fig. 1). Se contabilizaron 493 presas, las que fueron clasificadas en 40 categorías taxonómicas (Cuadro 2). Las presas más importantes en su dieta fueron Araneae (IRI\%=34.96), Chironomidae $($ IRI $\%=33.08)$, Tipulidae $($ IRI $\%=11.44)$ y Gryllidae (IRI\%=7.31). Los restantes ítems alimentarios fueron considerados como presas de consumo secundario debido al bajo valor alcanzado con éste índice. El número medio de presas consumidas por todos los ejemplares de H. pulchellus fue $4.74 \pm 5.42$ y el volumen promedio de $75.08 \pm 68.45 \mathrm{~mm}^{3}$. Las hembras de $H$. pulchellus poseían en sus tractos digestivos un promedio de $5.16 \pm 6.27$ presas, con un volumen promedio de $79.69 \pm 111.99 \mathrm{~mm}^{3}$ por gramo del ejemplar. En tanto, en los machos el promedio fue de $4.71 \pm 5.40$ presas, y el volumen promedio de $74.80 \pm 65.79 \mathrm{~mm}^{3}$. Se encontró una variación mensual en el número de presas consumidas, siendo julio el mes diferente (Fisher, $\mathrm{p}<0.05$ ). 
CUADRO 1

Análisis (ANCOVAs) de los cuerpos grasos y de la cantidad y volumen de las presas consumidas entre sexos, entre meses y entre especies (en $H$. pulchellus no se compararon los sexos debido al reducido número de hembras)

TABLE 1

Analysis (ANCOVAs) of body fat and the amount and volume of prey consumed between sexes, between months and between species (in $H$. pulchellus between sex comparisons were skip over due to the few females)

\begin{tabular}{|c|c|c|c|}
\hline Variables & $\mathrm{F}$ & Tamaño de muestra & $\mathrm{p}$ \\
\hline \multicolumn{4}{|c|}{ H. pulchellus } \\
\hline $\mathrm{CG}_{x^{2}-0.5}$ mensual & 0.331 & $\operatorname{may}=26 ; j u l=24 ;$ ago $=19 ; \mathrm{sep}=11$ & 0.857 \\
\hline Vol. mensual & 2.175 & may $=27 ;$ jul $=29 ;$ ago $=29 ;$ sep $=17$ & 0.096 \\
\hline $\mathrm{N}_{\log (x+1)}$ mensual & 5.215 & $\operatorname{may}=27 ; j u l=29 ;$ ago $=29 ; \mathrm{sep}=17$ & 0.002 \\
\hline \multicolumn{4}{|c|}{ D. nanus } \\
\hline $\mathrm{CG}+\hat{0}$ & 4.357 & $q=59 ; \hat{\jmath}=55$ & 0.039 \\
\hline Vol q̊ํ & 0.550 & $q=50 ; \widehat{\jmath}=43$ & 0.460 \\
\hline $\mathrm{N}+\widehat{+}$ & $<0.001$ & $q=50 ; \jmath^{\lambda}=43$ & 0.990 \\
\hline CG mensual & 1.83 & may $=29 ;$ jun $=29 ;$ jul $=27 ;$ ago $=28$ & 0.146 \\
\hline Vol. mensual & 0.963 & may $=24 ;$ jun $=29 ;$ jul $=21 ;$ ago $=18$ & 0.414 \\
\hline $\mathrm{N}$ mensual & 0.72 & may $=24 ;$ jun $=29 ;$ jul $=21 ;$ ago $=18$ & 0.541 \\
\hline \multicolumn{4}{|c|}{ H. pulchellus y D. nanus } \\
\hline CG & 9.524 & $\mathrm{Hp}=81 ; \mathrm{Dn}=114$ & 0.002 \\
\hline $\mathrm{Vol}_{\log (x+1)}$ & 13.83 & $\mathrm{Hp}=104 ; \mathrm{Dn}=93$ & $<0.001$ \\
\hline $\mathrm{N}_{\log (x+1)}$ & 6.87 & $\mathrm{Hp}=104 ; \mathrm{Dn}=93$ & 0.011 \\
\hline
\end{tabular}

(CG) peso de los cuerpos grasos, (N) número de presas consumidas, (Vol) volumen de las presas consumidas, Hp: $H$. pulchellus, Dn: D. nanus. Co-variable: peso del ejemplar. Para cumplir con los requisitos del ANCOVA, en los casos en los que fue necesario los datos fueron transformados siguiendo las fórmulas: $\log (\mathrm{x}+1)$ o $\mathrm{x}^{2}-0.5$.

En D. nanus, de los 114 ejemplares analizados 93 contenían alimentos. Se observó una mayor cantidad de tractos gastrointestinales vacíos en los meses invernales de julio y agosto (Fig. 1). Se contabilizaron 326 presas, las que fueron clasificadas en 39 categorías taxonómicas (Cuadro 2). Las presas más importantes en su dieta fueron Chironomidae $($ IRI\%=48.14), Tipulidae (IRI\%=18.41), Psychodidae $($ IRI\%=7.44) y Araneae $($ IRI\% $=7.34)$. El número medio de presas consumidas por todos los ejemplares de D. nanus fue $3.51 \pm 2.58$, y el volumen promedio de $8.15 \pm 2.58 \mathrm{~mm}^{3}$. Las hembras de $D$. nanus presentaron en sus tractos digestivos un promedio de $3.70 \pm 2.60$ presas, con un volumen promedio de $9.95 \pm 10.47 \mathrm{~mm}^{3}$. En tanto, en los machos el promedio fue de
$3.27 \pm 2.58$ presas, y el volumen promedio de $5.41 \pm 5.16 \mathrm{~mm}^{3}$. Los resultados de los ANCOVAs sobre la variación entre sexos de ambas especies, entre meses y entre especie del Vol y $\mathrm{N}$ de presas se expresan en el cuadro 1.

La diversidad de contenidos gastrointestinales esperada según la curva de rarefacción para 93 ejemplares fue 2.53 en $H$. pulchellus y 2.91 en $D$. nanus. La equitatividad en $H$. pulchellus fue de 0.69 y la riqueza de 40 categorías presa. En cambio, la equitatividad en $D$. nanus fue de 0.79 y la riqueza de 49 categorías presa. El solapamiento del nicho trófico observado entre $H$. pulchellus y $D$. nanus fue elevado ( $\mathrm{Ojk}=0.78)$ y superior al esperado por azar (media de los índices simu- 

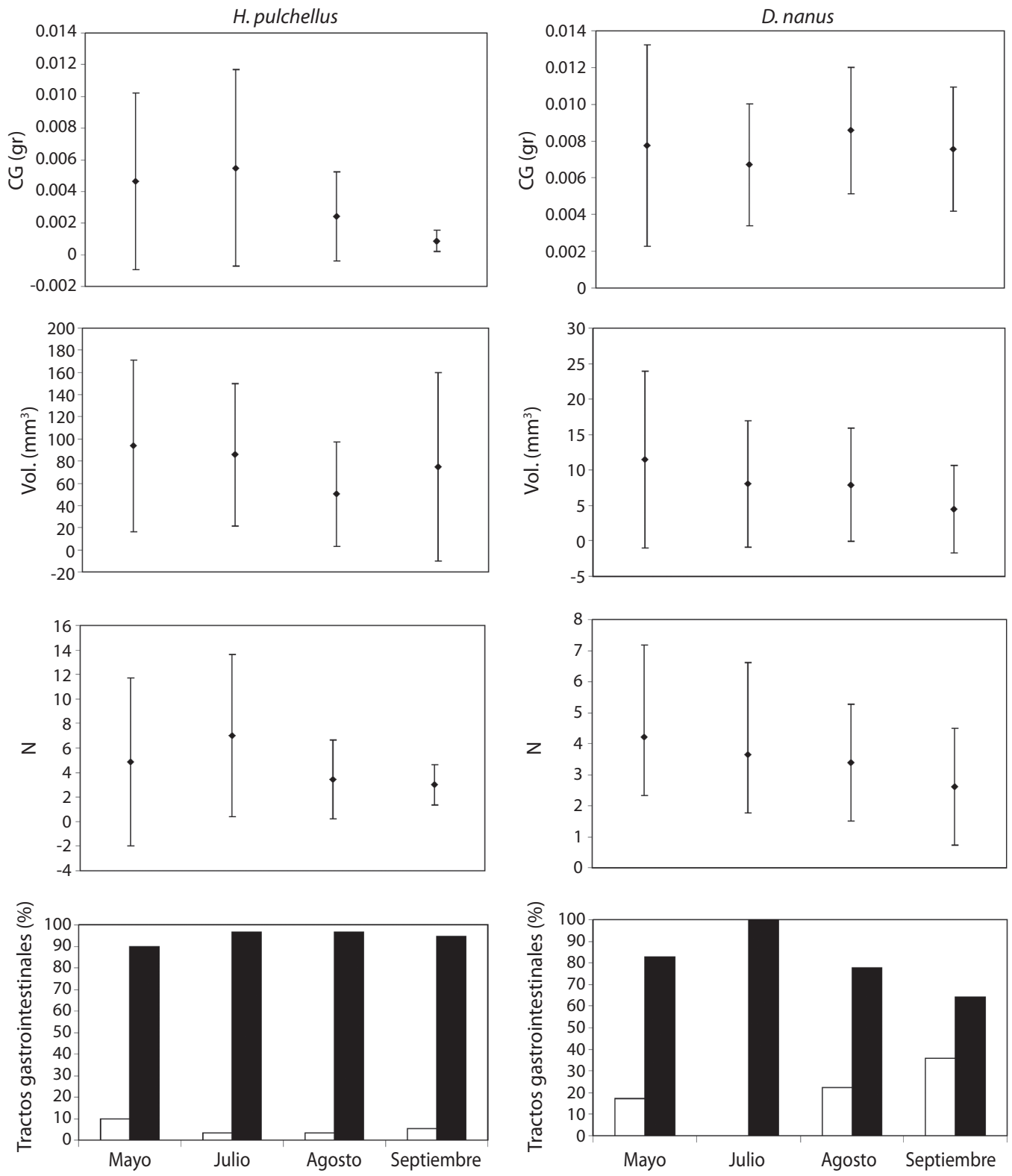

Fig. 1. Variación mensual de los cuerpos grasos, volumen y número de presas consumidas y porcentaje de tractos gastrointestinales con (barra negra) y sin (barra blanca) presas para cada especie (en H. pulchellus no se incluyó el mes de junio debido a que solo se contaba con un solo ejemplar y en D. nanus se excluyó septiembre, donde se disponía de dos ejemplares).

Fig. 1. Monthly variation of fat bodies, volume and number of prey consumed and percentage of gastrointestinal tracts with (black) and without (white) preys in each species (we exclude from figure the only H. pulchellus captured on June and the two D. nanus captured on September). 
CUADRO 2

Valores de la dieta de H.pulchellus y D. nanus

TABLE 2

Diet values of $H$. pulchellus and D. nanus

\begin{tabular}{|c|c|c|c|c|c|c|c|c|}
\hline \multirow{2}{*}{ Categorías presa } & \multicolumn{4}{|c|}{ H. pulchellus } & \multicolumn{4}{|c|}{ D. nanus } \\
\hline & $\mathrm{N} \%$ & $\mathrm{~F} \%$ & V\% & IRI $\%$ & $\mathrm{~N} \%$ & $\mathrm{~F} \%$ & V\% & IRI\% \\
\hline Insecta & - & - & - & - & 0.30 & 1.08 & 0.01 & 0.01 \\
\hline Insecta larva s/d & 0.41 & 1.92 & 0.63 & 0.05 & 0.30 & 1.08 & 0.61 & 0.03 \\
\hline \multicolumn{9}{|l|}{ Collembola } \\
\hline Entomobridae & - & - & - & - & 4.48 & 12.90 & 0.42 & 1.90 \\
\hline Poduridae & 5.88 & 3.85 & 0.01 & 0.57 & 0.90 & 2.15 & 0.01 & 0.06 \\
\hline Sminthuridae & 0.41 & 1.92 & 0.00 & 0.02 & 0.90 & 2.15 & 0.01 & 0.06 \\
\hline \multicolumn{9}{|l|}{ Orthoptera } \\
\hline Acrididae & 0.20 & 0.96 & 1.49 & 0.04 & - & - & - & - \\
\hline Gryllidae & 4.26 & 15.38 & 14.55 & 7.31 & - & - & - & - \\
\hline Tettigonidae & 0.61 & 2.88 & 5.23 & 0.43 & - & - & - & - \\
\hline \multicolumn{9}{|l|}{ Dermaptera } \\
\hline Dermaptera adulto $\mathrm{s} / \mathrm{d}$ & 1.22 & 5.77 & 1.72 & 0.43 & - & - & - & - \\
\hline \multicolumn{9}{|l|}{ Heteroptera } \\
\hline Heteroptera adulto s/d & - & - & - & - & 0.90 & 3.23 & 0.42 & 0.13 \\
\hline Heteroptera larva s/d & 0.20 & 0.96 & 0.04 & 0.01 & 0.30 & 1.08 & 0.07 & 0.01 \\
\hline Auchenorrihyncha s/d & 0.41 & 1.92 & 0.61 & 0.05 & 0.30 & 1.08 & 6.00 & 0.20 \\
\hline Aphididae & 0.20 & 0.96 & 0.00 & 0.00 & 2.39 & 2.15 & 1.13 & 0.23 \\
\hline Cercopidae & 1.62 & 6.73 & 1.05 & 0.45 & 4.48 & 9.68 & 2.89 & 2.15 \\
\hline Cicadellidae & 1.62 & 5.77 & 3.21 & 0.70 & 1.19 & 4.30 & 1.00 & 0.28 \\
\hline Lygaeidae & - & - & - & - & 0.30 & 1.08 & 0.21 & 0.02 \\
\hline Miridae & 0.20 & 0.96 & 0.04 & 0.01 & 0.60 & 2.15 & 0.92 & 0.10 \\
\hline Psyliidae & - & - & - & - & - & - & - & - \\
\hline \multicolumn{9}{|l|}{ Odonata } \\
\hline Coenagrionidae & 0.41 & 0.96 & 0.63 & 0.03 & - & - & - & - \\
\hline Anisoptera larva s/d & 0.20 & 0.96 & 0.85 & 0.03 & - & - & - & - \\
\hline \multicolumn{9}{|l|}{ Lepidoptera } \\
\hline Lepidoptera adulto s/d & 0.61 & 2.88 & 0.56 & 0.09 & - & - & - & - \\
\hline Lepidoptera larva s/d & 0.61 & 2.88 & 4.43 & 0.37 & 1.19 & 1.08 & 3.08 & 0.14 \\
\hline \multicolumn{9}{|l|}{ Coleoptera } \\
\hline Coleoptera larva s/d & 2.03 & 4.81 & 7.55 & 1.16 & - & - & - & - \\
\hline Carabidae & 2.84 & 11.54 & 3.06 & 1.72 & 1.79 & 5.38 & 1.40 & 0.52 \\
\hline Coccinillidae & 1.83 & 7.69 & 9.19 & 2.14 & - & - & - & - \\
\hline Curculionidae & 0.61 & 1.92 & 0.59 & 0.06 & - & - & - & - \\
\hline Elateridae & 0.20 & 0.96 & 0.20 & 0.01 & - & - & - & - \\
\hline Hydrophilidae & 0.20 & 0.96 & 1.61 & 0.04 & 0.01 & 0.01 & 0.20 & 0.00 \\
\hline Lampyridae & 0.20 & 0.96 & 0.94 & 0.03 & - & - & - & - \\
\hline Staphilinidae & 0.20 & 0.96 & 0.28 & 0.01 & - & - & - & - \\
\hline \multicolumn{9}{|l|}{ Hymenoptera } \\
\hline Hymenoptera adulto s/d & 0.20 & 0.96 & 0.06 & 0.01 & - & - & - & - \\
\hline Braconidae & - & - & - & - & 0.30 & 1.08 & 0.09 & 0.01 \\
\hline Diapriidae & - & - & - & - & 0.60 & 2.15 & 0.07 & 0.04 \\
\hline Formicidae & 2.03 & 8.65 & 1.04 & 0.67 & 0.90 & 3.23 & 0.41 & 0.13 \\
\hline Ichneumonidae & 0.61 & 2.88 & 0.48 & 0.08 & 0.30 & 1.08 & 0.59 & 0.03 \\
\hline
\end{tabular}


CUADRO 2 (Continuación)

Valores de la dieta de H.pulchellus y D. nanus

TABLE 2 (Continued)

Diet values of $H$. pulchellus and D. nanus

\begin{tabular}{|c|c|c|c|c|c|c|c|c|}
\hline \multirow{2}{*}{ Categorías presa } & \multicolumn{4}{|c|}{ H. pulchellus } & \multicolumn{4}{|c|}{ D. nanus } \\
\hline & N\% & $\mathrm{F} \%$ & $\mathrm{~V} \%$ & IRI $\%$ & $\mathrm{~N} \%$ & $\mathrm{~F} \%$ & $\mathrm{~V} \%$ & IRI $\%$ \\
\hline \multicolumn{9}{|l|}{ Diptera } \\
\hline Diptera adulto s/d & 0.41 & 1.92 & 0.04 & 0.02 & 2.99 & 7.53 & 3.99 & 1.58 \\
\hline Diptera larva s/d & - & - & - & - & 0.30 & 1.08 & 0.06 & 0.01 \\
\hline Diptera pupa s/d & 0.20 & 0.96 & 0.79 & 0.02 & - & - & - & - \\
\hline Bibionidae & - & - & - & - & 0.30 & 1.08 & 2.22 & 0.08 \\
\hline Cecidomyiidae & - & - & - & - & 0.30 & 1.08 & 0.01 & 0.01 \\
\hline Ceratopogonidae & 1.42 & 5.77 & 0.07 & 0.22 & 1.79 & 2.15 & 0.83 & 0.17 \\
\hline Chironomidae & 33.87 & 27.88 & 13.10 & 33.08 & 19.10 & 29.03 & 35.97 & 48.14 \\
\hline Chloropidae & 0.61 & 0.96 & 0.03 & 0.02 & - & - & - & - \\
\hline Culicidae & 1.62 & 6.73 & 0.35 & 0.34 & 2.99 & 6.45 & 4.24 & 1.40 \\
\hline Dixidae & - & - & - & - & 0.30 & 1.08 & 0.11 & 0.01 \\
\hline Dolichopodidae & - & - & - & - & 2.39 & 5.38 & 1.22 & 0.58 \\
\hline Ephidridae & - & - & - & - & 0.30 & 1.08 & 0.07 & 0.01 \\
\hline Muscidae & 1.62 & 6.73 & 0.41 & 0.35 & 4.78 & 13.98 & 6.30 & 4.66 \\
\hline Psychodidae & - & - & - & - & 11.04 & 20.43 & 1.06 & 7.44 \\
\hline Sarcophagidae & 0.20 & 0.96 & 0.30 & 0.01 & - & - & - & - \\
\hline Sciaridae & - & - & - & - & 1.19 & 4.30 & 0.70 & 0.24 \\
\hline Sphaeroceridae & - & - & - & - & 3.58 & 7.53 & 1.44 & 1.14 \\
\hline Tephritidae & - & - & - & - & 0.60 & 2.15 & 1.09 & 0.11 \\
\hline Tipulidae & 11.36 & 33.65 & 2.10 & 11.44 & 10.75 & 25.81 & 12.95 & 18.41 \\
\hline \multicolumn{9}{|l|}{ Arachnida } \\
\hline Acari & 3.04 & 7.69 & 0.01 & 0.59 & 5.97 & 13.98 & 0.08 & 2.54 \\
\hline Araneae & 12.98 & 49.04 & 15.25 & 34.96 & 5.67 & 20.43 & 6.25 & 7.34 \\
\hline \multicolumn{9}{|l|}{ Malacostraca } \\
\hline Isopoda & 2.64 & 9.62 & 7.48 & 2.46 & 0.30 & 1.08 & 1.87 & 0.07 \\
\hline
\end{tabular}

$(\mathrm{N} \%)$ porcentuales de numerosidad, $(\mathrm{F} \%)$ frecuencia de ocurrencia, (V\%) volumen e (IRI\%) Índice de Importancia Relativa jerarquizado de las presas, s/d: sin determinar.

lados: $\mathrm{Ojk}=0.04 ; \mathrm{p}[\mathrm{observed} \geq$ expected $]<0.01 ; \quad$ diversidad, equitatividad y riqueza de presas $\mathrm{p}[$ observed $\leq$ expected $]=1$ ).

\section{DISCUSIÓN}

En la planicie aluvial del río Paraná Medio, tanto H. pulchellus como D. nanus se alimentaron durante la temporada de bajas temperaturas. Se observó que ambas especies consumieron en común varios tipos de presa, principalmente algunas familias de dípteros (quironómidos y tipúlidos). Esto condujo al elevado solapamiento del nicho trófico y la similitud entre la encontradas. Si bien no se realizó una comparación con la oferta ambiental de presas, el gran consumo de dípteros, principalmente adultos de quironómidos, se explicaría por la abundancia y diversidad de estos insectos durante la estación de bajas temperaturas en los ambientes de la planicie aluvial del río Paraná Medio (Montalto \& Paggi 2006, Zilli et al. 2008). En H. pulchellus, se concuerda con lo obtenido por Maneyro \& da Rosa (2004) quienes mencionan a las arañas y dípteros, como las presas más importantes en la dieta de esta especie durante la temporada fría en Uruguay. 
Por otro lado, al comparar la dieta de estas especies entre la temporada fría aquí analizada y las temporadas cálidas respecto a otros trabajos, se observó que los dípteros siguen siendo el ítem presa más importante (Basso1990, Maneyro \& da Rosa 2004).

Por otra parte, se ha demostrado que el volumen y número de presas consumidas se encuentran relacionados con el tamaño del depredador. La diferencia entre las dos especies en la cantidad y volumen de presas en los contenidos gastrointestinales, refleja la incorporación de presas más numerosas y voluminosas por parte de H. pulchellus, lo que probablemente se relacione con su mayor tamaño corporal y ancho de boca (López et al. 2005, da Rosa et al. 2011) e implique una optimización de la actividad de forrajeo.

Las bajas temperaturas de la estación de otoño-invierno no afectaría por igual a todas las especies de anuros de la planicie aluvial del Paraná, ya que, a diferencia de lo ocurrido en $D$. nanus, se encontró que $H$. pulchellus posee contenidos en casi todos los tractos digestivos analizados durante toda la estación fría. En esta especie se hallaron los cuerpos grasos poco desarrollados durante el período otoño-invierno evaluado y sólo los machos poseían algún grado de desarrollo de los cuerpos grasos. Esto podría deberse a que en las hembras los cuerpos grasos exhiben tamaño mínimo durante el período de desove (Díaz-Páez \& Ortiz 2001). Teniendo en cuenta que $H$. pulchellus se reproduce en invierno (Basso 1990, Peltzer \& Lajmanovich 2007, López et al. 2011), el mayor desarrollo de los cuerpos grasos en los machos se relacionaría con las actividades energéticamente demandantes que se encuentran realizando éstos durante la temporada de apareamiento (establecer los sitios de canto, defender estos sitios de machos competidores, emitir el canto reproductivo para atraer a las hembras potenciales para el apareamiento) (Wells 2007, Navas et al. 2008, Quiroga \& Sanabria 2012). En una población del sur de Brasil, Solé \& Pelz (2007) encontraron que la mayor parte de los machos de $H$. pulchellus tenían presas en el estómago mientras se encontraban en el lugar de reproducción y sugirieron que existe una gran congruencia de los hábitats de alimentación y apareamiento. El extremadamente bajo índice de vacuidad encontrado en los machos de H. pulchellus de la Isla Sirgadero y Reserva Universitaria concuerda con las observaciones realizadas por estos autores. Entonces, durante la prolongada temporada de canto reproductivo de esta especie (Basso 1990, Peltzer \& Lajmanovich 2007), los machos se valdrían en parte de sus reservas lipídicas y en parte de las presas ingeridas para sustentar esa actividad energéticamente demandante.

Por otra parte, el período de actividad de las especies suele ser más prolongado que el reproductivo (Vaira 2002, López et al. 2011) e implica, entre otras cuestiones, el tiempo dedicado a la recuperación y acumulación de reservas energéticas mediante la alimentación (Duellman \& Trueb 1986). En este sentido, a diferencia de $H$. pulchellus, D. nanus posee un pico de actividad y reproducción concentrado en la primavera-verano (Basso 1990, Peltzer \& Lajmanovich 2007, Sánchez et al. 2007), por lo que los ejemplares analizados para la temporada de bajas temperaturas no se encontrarían sometidos a los requerimientos energéticos de la actividad reproductiva. Esto contribuiría a explicar el mayor número de tractos digestivos vacíos hallados en los ejemplares de D. nanus activos durante el otoño-invierno. Además, las bajas temperaturas implicarían una restricción fisiológica para la actividad de forrajeo de esta especie (Wells 2007). Existen diversos estudios que han intentado dilucidar el bajo consumo de los anuros en estaciones frías, sugiriendo: a) un ritmo más lento del paso de alimentos a través del sistema digestivo (Lillywhite et al. 1973); b) una disminución en la tasa de ingesta (Lillywhite et al. 1973); c) un movimiento más lento de las presas que podría afectar la respuesta de alimentación de los anuros depredadores (Ewert 1987); o $d$ ) una disminución rápida en las tasas metabólicas, por la cual los anfibios que pasan el invierno pueden sobrevivir durante muchos meses sin alimento (Koskela \& Pasanen 1974, Morton 1981, López Jurado 1982). 
A diferencia de lo ocurrido con los contenidos gastrointestinales, se encontró que $D$. nanus posee sus cuerpos grasos bien desarrollados durante la temporada fría. Esto sugiere que, como la mayoría de los anfibios de climas templados (Feder \& Burggren 1992), los ejemplares de $D$. nanus que se encuentra activos durante estos meses fríos, utilizarían sus reservas lipídicas acumuladas durante la temporada cálida anterior para pasar el invierno. Cabe destacar que en las hembras de D. nanus, de mayor tamaño que los machos, se observó una acumulación superior de reservas en forma de cuerpos grasos. Este aumento post reproductivo en el tamaño de los cuerpos grasos, así como la diferencia entre sexos en la acumulación de reservas energéticas también fueron encontradas en otras especies de anuros de climas templados (Quiroga \& Sanabria 2012). La inversión energética en la producción de óvulos es muy elevada (Wells 2007), debido a esto las hembras de D. nanus necesitarían de una fuerte acumulación de reservas lipídicas para afrontar la maduración gonadal y encontrarse preparadas para el evento reproductivo de la próxima temporada (en primavera-verano; Basso 1990, Peltzer \& Lajmanovich 2007, Sánchez et al. 2007).

Los resultados del presente trabajo permiten sugerir que existen diferentes estrategias energéticas según el período reproductivo y para mantener la actividad durante la temporada de bajas temperaturas en cada especie. Estas diferencias, aunque más leves, también se registraron entre sexos dentro de cada especie. Por un lado, $H$. pulchellus continúa alimentándose aún con temperaturas bajas (dentro del espectro de temperaturas que se registran en la planicie aluvial del río Paraná Medio), y esta sería una forma de mantener el elevado gasto energético que implica la reproducción concentrada en los meses de otoño-invierno (Peltzer \& Lajmanovich 2007). Los cuerpos grasos proveerían una fuente extra de energía para mantener las exigencias del cortejo en los machos. Por otro lado, la actividad de alimentación de D. nanus durante esta estación de bajas temperaturas fue ostensiblemente menor que la de $H$. pulchellus, pero sus cuerpos grasos se encontraron bien desarrollados, por lo que principalmente obtendrían la energía para pasar el otoño-invierno y llegar en buenas condiciones a la temporada reproductiva en primavera-verano con éstas acumulaciones lipídicas (Peltzer \& Lajmanovich 2007, Quiroga \& Sanabria 2012). Finalmente, sería interesante que en estudios futuros se integraran las variables energéticas y dietéticas de otras especies para comprender y predecir mejor sus estrategias y comportamiento.

\section{AGRADECIMIENTOS}

El presente estudio forma parte del trabajo de la tesina de Licenciatura de Carolina E. Antoniazzi. Los autores agradecen a Romina Ghirardi y Pablo A. Scarabotti por su colaboración en los muestreos y distintas etapas de la realización del presente trabajo. A Danilo Demonte, Andrea Previtali y Andrés M. Attademo por las sugerencias realizadas sobre la versión presentada como tesina de licenciatura. A Eduardo Schaefer por las sugerencias y aportes en el trabajo. A tres revisores anónimos por los aportes en el manuscrito.

\section{RESUMEN}

Existe escasa información sobre la alimentación de las especies de anfibios activas durante la estación fría o las estrategias de utilización de reservas lipídicas como fuente de energía durante el periodo de bajas temperaturas. Por lo tanto, se analizaron las estrategias de obtención y acumulación energética durante la estación de bajas temperaturas en un anfibio que se reproduce principalmente en otoño-invierno, Hypsiboas pulchellus $(\mathrm{Hp})$, y uno que lo hace en primavera-verano, Dendropsophus nanus (Dn). Para ello se analizó la alimentación de ambas especies y se utilizó el peso de los cuerpos grasos (CG) como indicador del desarrollo de las reservas energéticas. Si bien se encontró una elevada similitud en la dieta entre las especies, esto no implicó la ausencia de diferencias en las estrategias de obtención energética entre ellas. El bajo índice de vacuidad gastrointestinal de $\mathrm{Hp}$, acompañado de CG poco desarrollados, permite señalar que, para sostener la elevada demanda energética de la actividad reproductiva concentrada en el otoño-invierno, esta especie continúa alimentándose aún en temperaturas bajas. Por otro lado, el índice de vacuidad de Dn fue mayor al de $\mathrm{Hp}$, mientras que 
sus CG se encontraron bien desarrollados, por lo que esta especie utilizaría en mayor medida sus reservas lipídicas como fuente de energía para transitar el período de bajas temperaturas y llegar en buenas condiciones a la temporada reproductiva en la próxima primavera-verano.

Palabras clave: Hypsiboas pulchellus, Dendropsophus nanus, dieta, cuerpos grasos, estación de bajas temperaturas, fuente de energía.

\section{REFERENCIAS}

Angulo, A., J.V. Rueda-Almonacid, J.V. Rodríguez-Mahecha \& E. La Marca (eds.). 2006. Técnicas de inventario y monitoreo para los anfibios de la región tropical andina. Conservación Internacional. Serie Manuales de Campo $\mathrm{N}^{\circ}$ 2. Panamericana Formas e Impresos S.A., Bogotá D.C., Colombia.

Basso, N. 1990. Estrategias adaptativas de una comunidad subtropical de anuros. Cuad. de Herp., Serie Monografías 1: 1-70.

Cuevas, M.F. \& R. Martori. 2007. Diversidad trófica de dos especies sintópicas del género Leptodactylus (Anura: Leptodactylidae) del sudeste de la provincia de Córdoba, Argentina. Cuad. de Herp. 21: 7-19.

da Rosa, I., A. Canavero, R. Maneyro \& A. Camargo. 2011. Trophic niche variation and individual specialization in Hypsiboas pulchellus (Duméril and Bibron, 1841) (Anura, Hylidae) from Uruguay. South Am. J. Herpetol. 6: 98-106.

Díaz-Páez, H. \& J.C. Ortiz. 2001. The reproductive cycle of Pleurodema thaul (Anura, Leptodactylidae) in central Chile. Amphibia-Reptilia 22: 431-445.

Duellman, W.E. \& L. Trueb. 1986. Biology of the Amphibia. Mcgraw-Hill Book Company, Nueva York, EEUU.

Dunham, A.E. 1983. Realized niche overlap, resource abundance and intensity of interspecific competition, p. 261-280. In R.D. Huey, E.R. Pianka \& T.W. Schoener (eds.). Lizards Ecology: studies of a model organism. Harvard University, Cambridge, Massachusetts, EEUU.

Duré, M.I. \& A.I. Kehr. 2004. Influence of microhabitat on the trophic ecology of two leptodactylids from northeastern Argentina. Herpetologica 60: 295-303.

Ewert, J.P. 1987. Neuroethology of releasing mechanisms: Prey catching in toads. Behav. Brain Sci. 10: 337-405.

Feder, M.E. \& W.W. Burggren. 1992. Environmental physiology of the amphibians. University of Chicago, Chicago, EEUU.

Gotelli, N.J. \& G.R. Graves. 1996. Null models in ecology. Smithsonian Institution Washington, D.C., EEUU.
Gotelli, N.J. \& G.L. Entsminger. 2003. Swap algorithms in null model analysis. Ecology 84: 532-535.

Gotelli, N.J. \& G.L. Entsminger. 2004. EcoSim: Null models software for ecology. Version 7.0. Acquired Intelligence Inc. \& Kesey-Bear, Jericho.

Guimarães, T.S., G.B. Figueiredo, D.O. Mesquita \& M.M. Vasconcellos. 2011. Ecology of Hypsiboas albopunctatus (Anura: Hylidae) in a Neotropical Savanna. J. Herpetol. 45: 244-250.

Iriondo, M.H., J.C. Paggi \& M.J. Parma (eds.). 2007. The Middle Paraná River: Limnology of a Subtropical Wetland. Springer, Heidelberg, Alemania.

Jørgensen, C.B. 1986. External and internal control of patterns of feeding, growth and gonadal function in a temperate zone anuran, the toad Bufo bufo. J. Zool. (London) 216: 211-241.

Jørgensen, C.B. 1992. Growth and reproduction. In M.E. Feder \& W.W. Burggren (eds.). Environmental physiology of the Amphibians. University of Chicago, Chicago, EEUU.

Koskela, P. \& S. Pasanen. 1974. The wintering of the common frog, Rana temporaria L., in northern Finland. Aquilo (Zool.) 15: 1-17.

Lillywhite, H.B., P. Licht \& P. Chelgren. 1973. The role of behavioral thermoregulation in the growth energetics of the toad, Bufo boreas. Ecology 54: 375-83.

López, J.A., P.M. Peltzer \& R.C. Lajmanovich. 2005. Dieta y solapamiento del subnicho trófico de nueve especies de leptodactílidos en el Parque General San Martín (Argentina). Rev. Esp. Herp. 19: 19-31.

López, J.A., R. Ghirardi, P.A. Scarabotti \& M.C. Medrano. 2007. Feeding ecology of Elachistocleis bicolor in a riparian locality of the middle Paraná River. Herp. J. 17: 48-53.

López, J.A., P.A. Scarabotti, M.C. Medrano \& R. Ghirardi. 2009. Is the red spotted green frog Hypsiboas punctatus (Anura: Hylidae) selecting its preys? The importance of prey availability. Rev. Biol. Trop. 57: 847-857.

López, J.A., P.A. Scarabotti \& R. Ghirardi. 2011. Seasonal patterns of abundance and recruitment in an amphibian assemblage from the Paraná River floodplain. Interciencia 36: 538-544.

López Jurado, L.F. 1982. Estudios sobre el sapo corredor (Bufo calamita) en el Sur de España. Doñana Act. Vert 9: 71-84.

Maneyro, R. \& I. da Rosa. 2004. Temporal and spatial changes in the diet of Hyla pulchella (Anura, Hylidae) in southern Uruguay. Phyllomedusa 3: 101-113.

Martori, R., L. Aun, A. Birri Rozzi, C. Giménez \& E. Heredia. 2005. Reproducción comparada de tres especies de anuros sintópicos de una localidad del sudeste de Córdoba. Cuad. de Herp. 18: 43-59. 
McDiarmid, R.W. 1994. Preparing amphibians as scientific specimens, p. 289-296. In W.R. Heyer, M.A. Donnelly, R.W. McDiarmid, L.C. Hayek \& M.S. Foster (eds.). Measuring and Monitoring Biological Diversity. Standard Methods for Amphibians. Smithsonian Institution, Washington D.C., EEUU.

Montalto, L. \& A.C. Paggi. 2006. Diversity of chironomid larvae in a marginal fluvial wetland of the Middle Paraná River floodplain, Argentina. Ann. Limnol. Int. J. Lim 42: 289-300.

Morton, M.L. 1981. Seasonal changes in total body lipid and liver weight in the Yosemite toad. Copeia 1981: 234-238.

Navas, C.A., Gomes F.R. \& J.E. Carvalho. 2008. Review: Thermal relationship and exercise physiology in anuran amphibians: integration and evolutionary implications. Comp. Biochem. Physiol. 51: 344-362.

Panigatti, J., J. Weber \& O. Pillati. 1981. Estado actual y futuro de los problemas de suelo de Santa Fe. INTA, Rafaela, Santa Fe, Argentina.

Parmelee, J.R. 1999. Trophic ecology of a tropical anuran assemblage. Scientific papers, Natural History Museum. Univer. Kansas 11: 1-59.

Peltzer, P.M. \& R.C. Lajmanovich. 2000. Dieta de Hyla nana (Anura: Hylidae) en charcas temporarias de la llanura aluvial del río Paraná, Argentina. Bol. Soc. Herp. Esp. 11: 71-73.

Peltzer, P.M. \& R.C. Lajmanovich. 2007. Amphibians, p. 341-360. In M.H. Iriondo, J.C. Paggi \& M.J. Parma (eds.). The Middle Paraná River. Limnology of a Subtropical Wetland. Springer-Verlag Berlin, Heidelberg, Alemania.

Pianka, E.R. 1973. The estructure of lizard communities. Ann. Rev. Ecol. Syst. 4: 53-74.

Pianka, E.R. 1974. Niche overlap and diffuse competition. PNAS 71: 2141-2145.

Pielou, E.C. 1966. The measurement of diversity in different types of biological collections. J. Theoret. Biol. 13: $131-144$

Pinkas, L., M.S. Oliphant \& Z.L. Iverson. 1971. Food habit of albacore bluefin, tuna and bonito in California water. California Department of Fishing Game. Fish. Bull. 152: 1105.

Prado, C.P.A. \& C.F.B. Haddad. 2005. Size-fecundity relationships and reproductive investment in female frogs in the Pantanal, South-Western Brazil. Herp. J. 15: 181-189.

Quiroga, L. \& E. Sanabria. 2012. Do reproductive parameters of Rhinella arenarum (Hensel, 1867) (Anura: Bufonidae) vary between the reproductive and postreproductive period?. Bel. J. Zool. 42: 68-73.

Sánchez, L.C., P.M. Peltzer, A.S. Manzano \& R.C. Lajmanovich. 2007. Dinámica de un ensamble de anuros en un humedal del tramo inferior del Río Paraná, Argentina Interciencia 32: 463-470.

Shannon, C.E. \& W. Weaver. 1949. The Mathematical Theory of Communications. University of Illinois, Urbana, Illinois, EEUU.

Solé, M. \& B. Pelz. 2007. Do male tree frogs feed during the breeding season? Stomach flushing of five syntopic hylid species in Rio Grande do Sul, Brazil. J. N. Hist. 41: 2757-2763.

Vaira, M. 2002. Anurans of a subtropical montane forest in northwestern Argentina: ecological survey and a proposed list of species of conservation concern. Biodiv. Conserv. 11: 1047-1062.

Vitt, L.J. \& R.D. Ohmart. 1975. Ecology, reproduction and reproductive effort of the iguanid lizard Urosaurus graciosus on the Lower Colorado River. Herpetologica 31: 56-65.

Wells, K.D. 2007. The Ecology and Behavior of Amphibians. The University of Chicago, Chicago, EEUU.

Wunder, B.A. 1984. Strategies for, and environmental cueing mechanisms of, seasonal changes in thermoregulatory parameters of small mammals, p. 165172. In J.F. Merritt (ed.). Winter Ecology of Small Mammals. Carnegie Museum of Natural History, Pittsburg, Pennsylvania, EEUU.

Zilli, F.L., L. Montalto \& M.R. Marchese. 2008. Benthic invertebrate assemblages and functional feeding groups in the Paraná River floodplain (Argentina). Limnologica 38: 159-171. 